Review

\title{
A Literature Review on Caching Transient Contents in Vehicular Named Data Networking
}

\author{
Marica Amadeo
}

check for updates

Citation: Amadeo, M. Literature

Review on Caching Transient Contents in Vehicular Named Data Networking. Telecom 2021, 2, 75-92. http:/ / doi.org/10.3390/ telecom2010006

Received: 23 December 2020 Accepted: 3 February 2021 Published: 9 February 2021

Publisher's Note: MDPI stays neutral with regard to jurisdictional clai$\mathrm{ms}$ in published maps and institutional affiliations.

Copyright: () 2021 by the author. Licensee MDPI, Basel, Switzerland. This article is an open access article distributed under the terms and conditions of the Creative Commons Attribution (CC BY) license (https:// creativecommons.org/licenses/by/ $4.0 /)$.
DIIES Department, University Mediterranea of Reggio Calabria, Via Graziella, 89124 Reggio Calabria, RC, Italy; marica.amadeo@unirc.it

\begin{abstract}
Vehicular Named Data Networking (VNDN) is a revolutionary information-centric architecture specifically conceived for vehicular networks and characterized by name-based forwarding and in-network caching. So far, a variety of caching schemes have been proposed for VNDN that work in presence of static Data packets, like traditional Internet contents. However, with the advent of Internet of Things (IoT) and Internet of Vehicles (IoV) applications, large sets of vehicular contents are expected to be transient, i.e., they are characterized by a limited lifetime and become invalid after the latter expires. This is the case of information related to road traffic or parking lot availability, which can change after a few minutes — or even after a few seconds-it has been generated at the source. The transiency of contents may highly influence the network performance, including the gain of in-network caching. Therefore, in this paper, we consider the dissemination of transient contents in vehicular networks and its effects on VNDN caching. By providing a detailed review of related work, we identify the main challenges and objectives when caching transient contents, e.g., to avoid cache inconsistency, to minimize the Age of Information (AoI) and the retrieval latency, and the main strategies to fulfill them. We scan the existing caching and replacement policies specifically designed for transient contents in VNDN and, finally, we outline interesting research perspectives.
\end{abstract}

Keywords: Vehicular Named Data Networking; vehicular networks; Named Data Networking; Information Centric Networking; Internet of Things

\section{Introduction}

Named Data Networking (NDN) is a revolutionary Information Centric Networking (ICN) architecture that turns the contents into the first class network citizens [1]. Instead of using IP addresses, NDN nodes directly leverage application-level content names to disseminate and retrieve self-consistent Data packets. In-network caching is natively enabled, which makes NDN a valuable solution for challenging wireless environments characterized by mobility and intermittent connectivity, like delay-tolerant and opportunistic networks and vehicular networks [2].

The applicability of NDN in vehicular environments has been originally presented in References [3,4]. There, the authors explored the benefits of name-based forwarding and caching provided by NDN against the host-centric connection-oriented IP-based model, which poorly suits highly dynamic environments with possibly opportunistic connectivity. The resulting architecture, referred to as Vehicular NDN (VNDN), is based on the broadcast exchange of content request packets, called Interests, and Data packets, which carry the requested contents. A vehicle or a Road Side Unit (RSU) acting as content consumer broadcasts an Interest to retrieve a named content. Any other node owing the requested Data packet, either the original producer or a cacher, can answer the request. Conversely, if the packet is not locally available, the node can further broadcast the Interest. Incoming Data packets can be potentially cached by any node to satisfy future requests. The storage unit, called Content Store (CS) in NDN, can be managed through different policies. Although the reference caching scheme is Cache Everything Everywhere (CEE) 
coupled with Least Recently Used (LRU) replacement, NDN is open to the implementation of other strategies.

So far, a large body of research has been devoted to the development of forwarding strategies that reduce potential broadcast storm issues and speed up the data retrieval in VNDN [5-7]. In parallel, several caching strategies have been proposed to improve the availability of contents [8,9]. However, these works have considered the dissemination of static contents, like traditional Internet multimedia files, which do not change over time. Vice versa, due to the increasing popularity of Internet of Things (IoT) and Internet of Vehicles (IoV) applications, a large number of vehicular contents are expected to be transient, i.e., they can change over time to reflect the current status of vehicles, streets, and nearby point of interests [10].

NDN nodes recognize transient contents, thanks to the so-called FRESHNESSPERIOD, a field in the header of the Data packet that can be set by the source to specify the lifetime of the content, in milliseconds (https: / / named-data.net/doc/NDN-packet-spec/current/ data.html, accessed on 23 December 2020). If a received Data packet carries a FRESHNESSPERIOD greater than zero, a node considers it fresh. The FRESHNESSPERIOD is treated as a timeout by the CS: when the latter expires, the packet is marked as non-fresh.

Clearly, the transiency of contents highly impacts on the performance of in-network caching and the research community is questioning if it would be still useful to fill the limited content store of NDN nodes with packets that are ready to expire. Especially in vehicular contexts, consumer applications are interested in fresh data. For instance, processing outdated information about road traffic conditions could compromise the reliability of intelligent driving applications. Receiving outdated information about parking lots availability may affect route and trip planning.

In this paper, we explore challenges and benefits of caching transient information in VNDN. As shown in Figure 1, after introducing the VNDN architecture, we develop a thorough analysis on caching transient contents by also exploring related research conducted over the years, outside the NDN scope. Then, we present an in-depth overview about how VNDN can improve its delivery performance if freshness-awareness is introduced in the caching and replacement mechanism. A set of lesson learned and interesting research perspectives are also outlined.

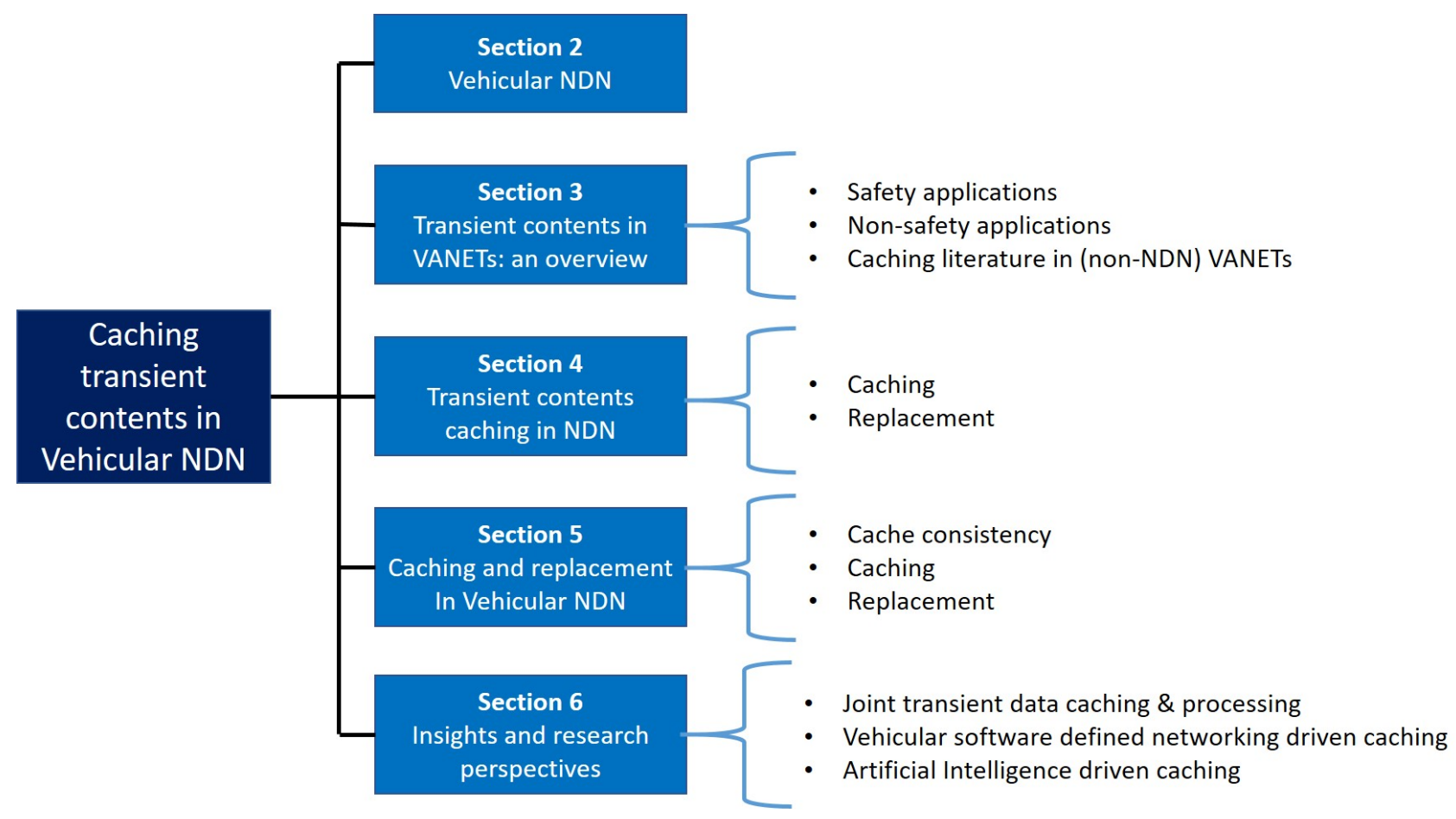

Figure 1. Organization of the paper (graphical view). 
A few existing survey papers about VNDN can be already found in the literature; however, none of them tackle our targets. In particular, the applicability of NDN in vehicular networks, coupled with a preliminary analysis of related work, was originally discussed in References [11,12]; however, caching aspects were only briefly introduced. The work in Reference [13] surveys existing NDN forwarding strategies for vehicular ad hoc networks (VANETs), without focusing on the literature about caching. A comprehensive and systematic survey of VNDN-based communications is reported in Reference [14], where the authors cover different architectural aspects related to naming, routing, forwarding, caching, mobility, and security. However, their analysis does not consider the case of transient information. Similarly, the work in Reference [15] is specifically devoted to VNDN caching, but it does not elaborate on the benefits and challenges of caching transient contents. To the best of our knowledge, this paper represents the first effort to review and analyze transient content caching in VNDN.

The rest of the paper is organized as follows. Section 2 introduces VNDN and the reference network scenario. Section 3 surveys existing works dealing with transient contents in traditional vehicular networks and identifies a set of lesson learned and inputs for the subsequent analysis in the NDN context, reported in Section 4. Section 5 surveys existing caching and replacement policies specifically designed for transient contents in VNDN, while Section 6 presents some future research perspectives. Finally, Section 7 concludes the paper.

\section{Vehicular NDN}

Vehicular networks were originally conceived to support two broad classes of applications, namely: (i) safety applications, devoted to improving the safety of drivers and passengers, and (ii) non-safety applications, with infotainment purposes. Today, however, the advent of intelligent and autonomous driving applications has turned connected vehicles into one of the major contributors of IoT traffic and has promoted the new Internet of Vehicle (IoV) paradigm. Provided with multiple on-board sensors, cameras and GPS devices, vehicles will generate and process terabytes of contents per driving hour, while offering advanced driving experiences to drivers and passengers. A proper network architecture is, therefore, required that effectively and reliably delivers vehicular information in presence of mobility, harsh propagation conditions, and typically stringent latency constraints. In this challenging context, NDN, and particularly its customization in the vehicular environment, namely VNDN [4], represents an extremely promising solution.

In principle, VNDN can operate in presence of multiple wireless access standards, including the most prominent alternatives for vehicular networks, namely IEEE 802.11p and Cellular Vehicle to Everything (C-V2X) [16]. In the following, however, we mainly refer to vehicular ad hoc networks (VANETs) based on IEEE 802.11p, which is the reference technology in almost all the VNDN-based solutions available in the literature [14].

As shown in Figure 2, in the considered VNDN network, vehicular on-board units (OBUs) interact among each other through vehicle-to-vehicle (V2V) communications, and with nearby road-side units (RSUs) and other infrastructure elements, e.g., smart semaphores, through vehicle-to-infrastructure (V2I) communications. In the majority of VNDN deployments, V2V and V2I interactions are based on the broadcast exchange of Interest and Data packets to maximize the data sharing over the wireless channel.

The basic forwarding strategy in VNDN consists of controlled-flooding [4]. A consumer node (either a vehicle or a RSU) broadcasts the Interest packet. Each node $R$ receiving the request checks if it maintains the Data packet in the CS. If so, it schedules the broadcasting of the Data after a certain time, to avoid collisions with other neighbors. Otherwise, $R$ schedules the re-transmission of the Interest packet. If $R$ overhears the Data or the Interest transmitted by other nodes, then it cancels its own transmission. In case of an Interest (re)-transmission, the request is maintained in a data structure called Pending Interest Table (PIT) until the correspondent Data packet is received or the Interest lifetime expires. 


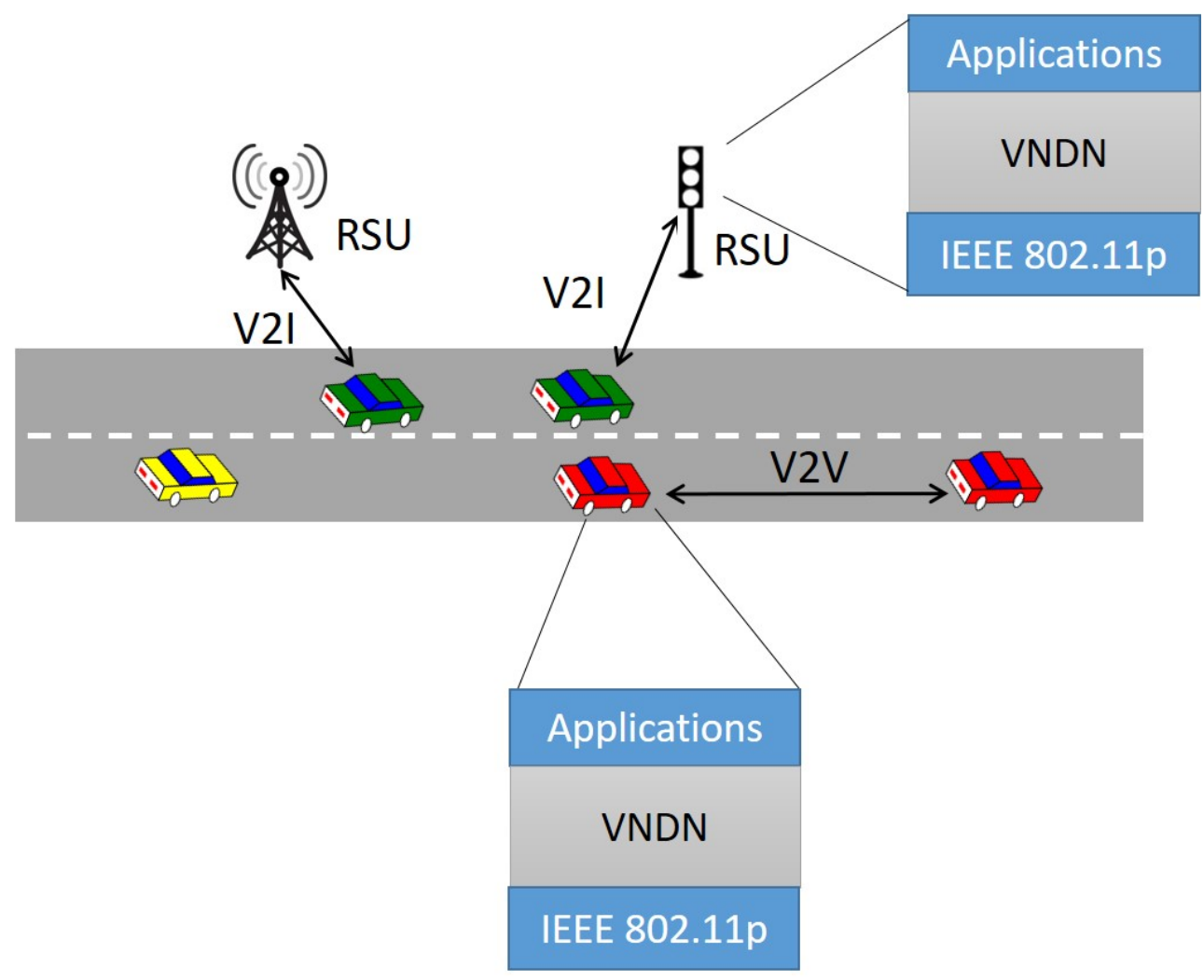

Figure 2. Reference Vehicular Named Data Networking (VNDN) scenario.

NDN nodes are usually provided with multiple access network interfaces; therefore, to forward the content requests, they record the outgoing interface(s) per each named prefix in the so-called Forwarding Information Base (FIB). In the considered scenario, however, we assume that node $R$ is provided with a single wireless access interface, i.e., IEEE 802.11p; therefore, it simply re-broadcasts the packet over the same interface the Interest arrived from. The request travels hop by hop in the network until the Data is discovered or an hop count, carried in the header and decremented at each hop, is set to zero.

Multiple approaches have been proposed over the years to limit the adverse effects of packet broadcasting, e.g., high traffic congestion and limited reliability, and improve the forwarding decision. For instance, in References [7,17], the effects of the hop count added in the Interest header to limit the multi-hop forwarding of the requests are studied. In Reference [18], controlled-flooding is used only to discover the content provider (either the original producer or a cacher). Then, subsequent Interests are marked with the MAC address of the discovered provider and only the nodes in the path towards it are allowed to forward the requests. In Reference [19], the authors leverage the GPS technology on-board of vehicles and extend VNDN forwarding with the concept of geo-location. Content name prefixes are mapped to the geographic areas of their producers and Interests are forwarded based on that geographical information. Vice versa, in Reference [20], vehicles maintain a table that tracks information about the neighbor nodes, like their speed and direction. When an Interest must be forwarded, vehicles access the table to select the best forwarder among their neighbors.

Notwithstanding the different implementation details of the forwarding strategy, all VNDN designs benefit from the native in-network caching function provided by NDN. The simplest and fast caching scheme, implemented in the vanilla NDN implementation, is CEE, where all incoming packets are cached. If the CS is full, a replacement operation is performed which can be based on traditional policies, like LRU.

Many other caching solutions were proposed in the literature that outperform CEE. They range from simple random caching [21] to topology-based and popularity-based schemes [14]. However, the majority of them refer to traditional static contents [22]. 


\section{Transient Contents in VANETs: An Overview}

Even before the advent of VNDN, the transmission of transient contents was considered in vehicular networks, and the majority of attention was devoted to the context of safety applications [23]. Indeed, these latter require the regular exchange of timely information about the state of vehicles, e.g., position and speed, to effectively protect the users on wheels. Sometimes simply referred to as beacons, different status messages that change over time have been standardized over the years. For instance, cooperative awareness messages (CAM) were defined by the European Telecommunication Standard Institute (ETSI) in the standard EN 302 637-2 [24]. Each CAM includes the current position, speed and direction of the originating vehicle and the transmission triggering conditions depend on the variation of the kinematics parameters. Basically, the vehicle compares its current kinematic measurements with the ones of the last generated CAM and, if the difference is above a pre-defined threshold, the vehicle triggers a new CAM transmission. Therefore, the actual CAM sending rate is generally unpredictable; ETSI only defines lower and upper bounds for the generation period, i.e., respectively, $100 \mathrm{~ms}$ and $1000 \mathrm{~ms}$. Of course, time-critical safety applications built on top of CAMs (e.g., for accident warning) are interested in receiving fresh information; otherwise, they cannot work properly.

In the following, we shortly introduce the main literature dealing with transient contents in vehicular networks, but outside the NDN context. We observe that the majority of research works in this field target the Age of Information (AoI) concept when considering safety applications and routing protocols and only few works were devoted to transient non-safety traffic. We conclude the section by identifying a set of insights for our subsequent analysis in VNDN networks.

\subsection{Minimizing Age of Information of Safety Information}

The first works in the context of transient contents for safety applications were devoted to minimize the average end-to-end delay observed in the vehicles' states within a certain group of nodes [23] and called into the picture the concept of AoI, a performance metric measuring information freshness at the destination node. By identifying the time elapsed since the current data was created, AoI identifies how outdated the retrieved information is.

We can assume that a content $c_{k}$ is updated over time by its source, which therefore originates a time series of content objects $c_{k, 1}, c_{k, 2}$, etc., each one characterized by a timestamp $T_{c_{k, 1}}, T_{c_{k, 2}}$, and so on. At time $t$, the age of $c_{k, 1}$ can be computed as: $A o I_{c_{k, 1}}=t-T_{c_{k, 1}}$. As shown in Figure 3, whenever a new message is received at the consumer, the AoI drops to zero, and then it grows until the next update.

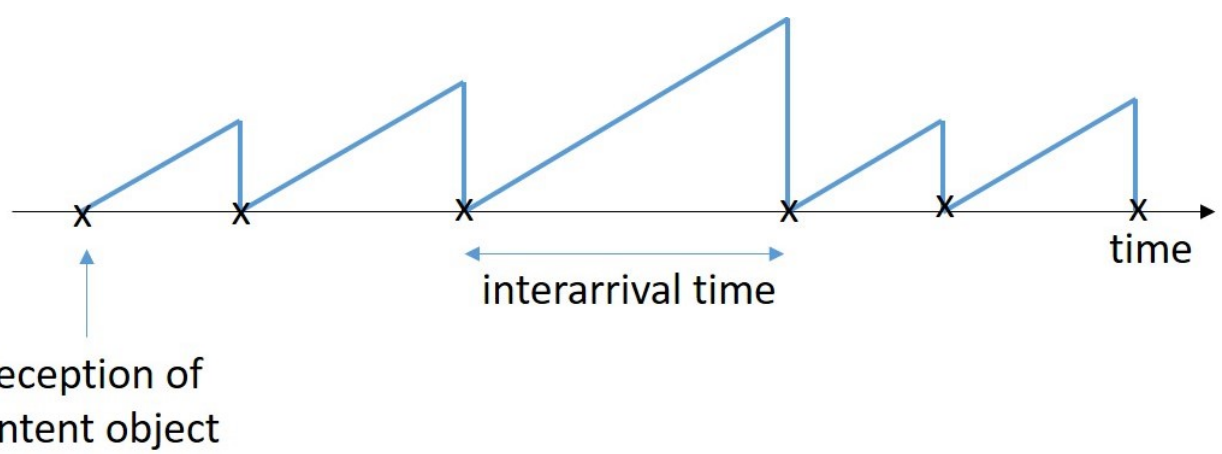

Figure 3. Age of Information (AoI) variation at the consumer side, when receiving subsequent updates of content $c_{k}$.

Ideally, each consumer should receive only the current valid version of the content [25]; information with high AoI is potentially unreliable and irrelevant. Receiving $c_{k, 1}$, when the most fresh version of the content is $c_{k, 2}$, could potentially compromise the behavior of the safety application. 
The preliminary work in Reference [26] analyzes the performance of information dissemination in urban VANETs with direct focus on AoI measures. The authors find that AoI grows almost linearly with the distance from the source and it also strongly depends on the density of vehicles. Similar considerations were reported in Reference [27], in the context of a free parking places discovery applications. In Reference [28], the authors focus on the AoI of broadcast beacons sent by vehicles and study the trade-off between the sending frequency of the messages and the congestion induced in the wireless IEEE 802.11p channel. An analytical model capturing the distributed contention in the VANET is proposed that shows how AoI changes with the beacon sending frequency and the vehicle density. When the latter parameters are high, the wireless medium tends to be congested, the channel access time increases, the message loss ratio increases due to collisions and the AoI increases, as well. On the other side, when the sending frequency reduces, the contention on the wireless channel reduces, as well, but the AoI intrinsically increases. Basically, the frequency of beacons should be adaptive and set according to the current vehicle density. Vehicles that move in a sparse zone can increase the message sending frequency, since the chances of collisions are low. Conversely, in crowded areas, they must decrease the sending frequency.

As a result, it is crucial to design transmission and caching mechanisms that minimize AoI and guarantee that the data, even when retrieved from the cache of an intermediate node, is not outdated. However, deciding when to discard stale data, and possibly update the cache, is not trivial in many circumstances. Sometimes, frequent updates are not possible because there are network constraints, like limited bandwidth [25], or because the cacher/recipient has lost the contact with the source or has poor and unreliable connectivity with it [26], e.g., a common condition in vehicular networks.

Literature works usually model the generation process of transient contents as a queuing system in which the packets arrive at the source by following a stochastic distribution [29]. The source transmits the packets using a certain policy, like First-Come-FirstServed (FCFS) or Least-Come-First-Served (LCFS), and only a single packet transmission occurs at a given time. For instance, in Reference [23], the authors assume that vehicles transmit their message updates periodically and find that AoI could be improved by controlling the MAC layer queue and, in particular, by changing the queuing policy from FCFS to LCFS. To further reduce the AoI, the work in Reference [30] proposes a proactive queue management scheme that discards old updates waiting in the queue when a new sample arrives.

More advanced scheduling mechanisms are proposed to minimize AoI and, sometimes, jointly satisfy other performance metrics, e.g., limiting the energy consumption or the network congestion [31]. More recently, the notion of AoI has been considered for analyzing ultra-reliable low-latency communications (URLLC) in 5G vehicular networks [32]. The proposal considers both periodic and stochastic arrivals of transient packets and allows vehicles to minimize their transmit power while ensuring URLLC communications based on a probabilistic measure of AoI violation.

\subsection{Transient Information for Non-Safety Applications and Routing Protocols}

Pushed by the increasing diffusion of IoT applications, vehicles are turning into mobile sensing platforms that capture transient information feeding a variety of smart city services, some of them with just infotainment purposes. Therefore, the interest in transient content is steadily growing also in the presence of non-safety applications, although they usually have less time critical requirements than safety applications. In addition to traditional Internet services and media sharing, they include air pollution monitoring, urban surveillance, waste management and many other location-based services [33]. Therefore, non-safety transient vehicular traffic can be roughly distinguished into two main classes: (i) small sized IoT data, e.g., captured by sensors deployed on-board of vehicles, and (ii) multimedia contents randomly or periodically updated, e.g., audio and video information about vehicular traffic captured by cameras. 
The work in Reference [34] considers generic transient contents that are updated at an origin Internet server at Poisson-distributed time instants and then distributed in the VANET through RSUs. Vehicles aim at maximizing the freshness of the retrieved content and the efficiency in the utilization of radio resources. To reach the targeted objectives, the proposal defines an optimal scheduling of data traffic over the existing links and selects a set of parked vehicles as optimal relays. Freshness information can be also included in the request/query packets [35]. If a newly received request is too old compared to other ones previously received, then the source or cacher node can simply decide to discard it.

In addition, multiple signaling packets originated and/or processed by vehicular routing protocols are transient information. In particular, geographic routing protocols leverage the (changing) geographic position of sender and receiver (usually obtained from GPS devices) to create path(s) between the endpoints [36]. Each sender employs a location-based service to discover the position of the destination and this information must be extremely fresh to ensure a reliable routing decision. Road-based routing protocols belong to this category: they create network routes as successions of road intersections where vehicles transit with high probability and provide multi-hop connectivity. The work in Reference [37] leverages real-time vehicular traffic information to create road-based paths in a proactive or reactive fashion. In the first case, vehicles generate connectivity packets $(\mathrm{CPs})$ periodically, by considering the estimated current number of vehicles in the networks, historical data about vehicle density, and the time elapsed from the last received $\mathrm{CP}$. The routing packets create a graph of connected road segments and are used to compute the shortest paths to destinations. Conversely, in the second case, routes are discovered on demand and reported in the packet headers to implement a source-routing scheme. In both cases, packets carry a timestamp to intrinsically reflect the freshness of the information. Synchronization among vehicles is reliably guaranteed by the GPS technology.

\subsection{Caching}

In-network caching in VANETs was considered years before the advent of VNDN, thanks to its intrinsic benefits, like reducing retrieval delay and traffic congestion and improving data access. However only few works investigated transient content caching and focused on two main research areas, namely the support of cache consistency and the design of caching decision strategies.

Cache consistency. In presence of transient contents and mobile environments, a first problem to face is the cache consistency, as preliminary analyzed in Reference [38]. The simplest way to ensure consistency is to include a lifetime in the content packet: when the latter expires, the node can drop the information since it is outdated. When the lifetime information is not available, then two approaches are possible: (i) in the source-initiated one, the source pushes to the other cachers the new version of the content; and (ii) in the cache-initiated one, the cachers pull the source to receive the update. To limit the signaling overhead between the endpoints, in Reference [38], some highly accessible and stable mobile nodes are selected to relay invalidation information to the cachers. A cooperative cache invalidation strategy is also proposed in Reference [39], where access points or RSUs are in charge of distributing updates of Internet contents. Clearly, these schemes are not always feasible in VANETs due to the presence of frequent disconnections and opportunistic contacts and the possible lack of infrastructure elements.

Caching strategies. A pioneering caching strategy for wireless ad hoc networks with data freshness awareness is Hamlet [35]. The proposal assumes that contents are fragmented in chunks tagged with a version identifier value, to distinguish the fresh ones from the stale ones. Consumers nodes cache packets according to a metric called content presence index, which estimates the availability of the content in the network. This information is obtained by taking into account the messages overheard in the neighborhood and the hop count distance from the origin source/cacher piggybacked in the retrieved content. In order to maximize the data diversity and limit the network traffic, the caching probability is inversely proportional to the presence index: there is basically no need to 
cache a content that is easily accessible in the network. In addition, if a node receives different versions of the requested content, it will only cache the most recent one and discard the others. If an older version is available in the cache, it will be replaced.

More recently, the work in Reference [40] defined a caching strategy based on the relationship between content popularity and freshness. Potential cachers track the AoI of contents and their request rate and select the content to cache in order to maximize the cache hit ratio. The rationale behind the conceived strategy is twofold: first, packets with higher AoI are less likely to be requested, and, second, popular packets have a higher chance to be requested. The strategy is autonomous and based on local measurements of content request rate and AoI.

\subsection{Main Insights}

As summarized in Table 1, our review shows that transient contents were considered in VANETs literature although less attention was devoted to the design of caching strategies.

Table 1. Transient Contents in (non-Named Data Networking (NDN)) vehicular ad hoc networks (VANETs): main topics and findings.

\begin{tabular}{|c|c|c|}
\hline Topic & Challenges & Main Findings \\
\hline AoI Assessment & $\begin{array}{l}\text { Vehicular applications, especially safety } \\
\text { ones, are interested in the freshest content } \\
\text { version }\end{array}$ & $\begin{array}{l}\text { AoI increases with the distance between } \\
\text { the endpoints and the vehicle } \\
\text { density }[26,27]\end{array}$ \\
\hline AoI Minimization & $\begin{array}{l}\text { - To reduce AoI when dealing with } \\
\text { transient safety and non-safety traffic }\end{array}$ & $\begin{array}{l}\text { - Smart queuing mechanisms at the MAC } \\
\text { layer, e.g., LCFS [23,29] } \\
\text { Timestamp in both query and content } \\
\text { packets to recognize and discard the older } \\
\text { ones [37] } \\
\text { To adapt the sending frequency to the } \\
\text { density of nodes }[28,31]\end{array}$ \\
\hline Cache Consistency & $\begin{array}{l}\text { - To recognize if the data is fresh or stale } \\
\text { To align cached contents with the updates } \\
\text { at the source }\end{array}$ & $\begin{array}{l}\text { Lifetime and timestamp/sequence } \\
\text { number information (if available) avoid } \\
\text { the issue }[37,38] \\
\text { Source-initiated pushing or } \\
\text { cache-initiated pulling to, respectively, } \\
\text { disseminate or receive the new content } \\
\text { version }[38,39]\end{array}$ \\
\hline Caching Strategy & $\begin{array}{l}\text { - To decide what to cache and for how long, } \\
\text { according to the freshness information, } \\
\text { among other parameters }\end{array}$ & $\begin{array}{l}\text { - To reduce the caching probability of } \\
\text { contents with high AoI [35,40] } \\
\text { If more versions of the content are } \\
\text { available, older ones should be } \\
\text { discarded [35] }\end{array}$ \\
\hline
\end{tabular}

Without loss of generality and despite the application domain (i.e., safety or not safety), we can broadly classify vehicular transient contents into two main categories:

- Contents with a fixed predefined lifetime. In this case, contents are characterized by a fixed lifetime that is usually application-specific [41]. Traditional beaconing approaches with a fixed frequency of message generation and monitoring applications that capture parameters at regular intervals belong to this category, e.g., environmental data, like humidity and temperature periodically captured by sensors deployed in RSUs or on-board of vehicles. Timestamp and lifetime (or refresh period) information can be set by the source and advertised directly in the content packet. Therefore it is extremely simple to maintain cache consistency and evict stale data. Thanks to GPS receivers, synchronization can be easily maintained among vehicles and infrastructure elements. 
- Contents with a variable lifetime. In this case, the lifetime is a-priori unknown: contents are updated by their producers randomly or on an event basis. Sometimes, the generation sampling rate is the result of policies that trade off between the timeliness of the information and other network parameters [31]. When different versions of the content are available in the network, a timestamp or a sequence number set by the source are crucial to recognize the freshest versions.

Related literature works have also identified some intrinsic features of vehicular communications that affect information freshness, namely the distance between the communication endpoints and the vehicle density. Queue management at the MAC layer has been recognized as a fundamental feature to reduce the age of information and different scheduling techniques have been explored. Vice versa, we could not find many solutions about freshness-aware caching. This is mainly due to the fact that the interest in transient contents is relatively new and mainly related to the advent of IoT/IoV applications and NDN. Moreover, NDN is the first popular networking architecture providing a reference in-network caching mechanism able to work in heterogeneous network environments, ranging from the wired Internet to vehicular and IoT networks.

\section{Transient Contents Caching in NDN}

The lifetime of transient contents can largely vary from some milliseconds to minutes or hours, depending on the application domain and the characteristics of the source. Therefore, the first research works in this field were centered to verify if NDN caching is still useful in presence of transient contents, especially those that expire very quickly, like in the context of IoT and wireless sensor networks [42,43]. Since these environments are part of the complex IoV vision, we provide a basic review of the main research results obtained in this area.

\subsection{Caching Policies in IoT and Sensor Networks}

In Reference [42], Baccelli et al., present a detailed experimentation of NDN performance in a smart campus in the presence of transient IoT contents. They find that caching is still extremely beneficial, especially in presence of multi-hop lossy channels and constrained sources.

Vural et al., in Reference [43], defines a probabilistic caching strategy for Internet content routers that trades off between IoT data lifetime and multihop communications. As also found in previous works on vehicular networks [23], AoI increases with the distance between consumer and producer; therefore, the freshness is maximum when the data is directly fetched from its producer and decreases when the packet moves away from it. The caching decision tries to balance freshness and communication costs.

But freshness is not the only crucial parameter to consider when dealing with transient contents. In particular, sensors are the principal sources of transient contents, which are generated as time series of readings, e.g., humidity, temperature, and $\mathrm{CO}_{2}$ emissions. Being usually battery-powered devices, energy efficiency becomes a major concern. Indeed, on the one side, caching generally reduces the network energy consumption and the load on the producers, since queries can be resolved closer to the consumers. On the other side, a caching operation incurs a certain energy consumption on the node, which has to access his memory resource and transmits the information on behalf of the original source. As a result, in the presence of constrained devices, caching could be selectively performed or even completely inhibited.

In Reference [44], sensors autonomously decide whether to cache the received packets, according to a composite probabilistic decision metric that takes into account the freshness of the item, the current battery level and the free storage space. The higher the freshness, the battery level and the free storage space, the higher the caching probability. Vice versa, the probability decreases. A cooperative strategy is instead proposed in Reference [45], where the constrained devices share among each other the load of answering to the content requests. Each node in the network periodically broadcasts its data to the neighbors, which 
cache them. As a result, some devices can sleep, while the others remain active and provide the contents.

Fortunately, vehicles do not have strict energy constraints and they can easily collect data from neighbor sensors. Of course, although they may leverage consistent storage resources, the number of cacheable contents is generally higher than the available storage space and some selective caching decisions should be performed.

A content parameter that traditionally is taken into account to filter the caching decision is the content popularity: reasonably, the caching gain can be maximized if the more requested contents are cached.

The work in Reference [46] designs an autonomous probabilistic caching strategy for IoT contents that jointly considers lifetime and popularity parameters. The FRESHNESSPERIOD carried in the NDN Data packets is used by the potential cacher to estimate if packets are long-lived and short-lived, while the rate of received requests is used to distinguish popular and non-popular contents. The strategy assumes that non-popular contents are never cached (i.e., the caching probability is set to zero), while popular long-lived contents are always cached (i.e., the caching probability is set to one). Finally, popular short-lived contents are cached with a probability that is directly proportional to the freshness of the content. The same parameters and caching logic are used in Reference [47], but the proposed mechanism is cooperative and depends on the position of the caching nodes, which are classified into three main categories. Root nodes are directly connected to the IoT sources, edge nodes are directly connected to the consumers, and the remaining ones are middle-level nodes. A different caching threshold is assumed that varies depending on the node's category: edge nodes have the smallest threshold in order to cache more data and reduce the retrieval delay. Vice versa, root nodes have the highest caching threshold and are supposed to cache only long-lasting contents. Moreover, the threshold is adapted according to the storage space and the number of incoming requests, as previously conceived in the popularity-based scheme in Reference [48]. The threshold reduces if the cacher has free storage space and the incoming request rate decreases, while it increases in the opposite case.

All in all, it can be observed that freshness and popularity are two crucial content parameters when dealing with caching transient contents. In addition, some attributes of the nodes, like storage space, topological position, and possible energy constraints, can be taken into account, as in traditional caching strategies for static Internet contents [49].

\subsection{Replacement Policies}

Traditional replacement policies, like LRU and LFU, can work also in presence of transient contents. However, the freshness may also drive the cache replacement operation. Contents that are ready to expire are reasonably the best candidates to be evicted from the CS, when the latter is full and a new packet should be cached. If packets carry lifetime and timestamp information, it is trivial to check when the packet must be dropped.

Thanks to the FRESHNESSPERIOD carried in each Data packet, NDN can natively recognize if a transient packet is stale. Indeed, the FRESHNESSPERIOD is treated like a timeout by the CS, and, when it expires, the packet is marked as non-fresh.

NDN consumers decide whether Interests can be answered by non-fresh Data packets, or not, by using the Interest MUSTBEFRESH field. However, some designs, like the ones in References [41,44], decide to immediately evict from the CS all the stale packets, thus freeing resources for new fresh information.

When the lifetime is not known in advance, forecasting methods can be developed to estimate, at the consumer/cacher side, when a new version of the content will be generated at the source [50]. For example, the Least Fresh First (LFF) policy in Reference [50] has been conceived for transient traffic originated by sensors and evicts the items that are supposed to be replaced soon based on forecasting of sensors-related future events. In order to predict the residual lifetime of the packet, LFF leverages an Autoregressive Moving Average (ARMA) model for time series forecasting. 


\section{To Cache and Replace Transient Contents in VNDN}

In this section, we present a classification and analysis of the existing solutions proposed in the context of VNDN caching. First, we focus on the problem of cache consistency, and then we present the literature about caching decision and replacement policies.

\subsection{Cache Consistency}

In principle, multiple versions of the same content may exist in VNDN networks. The work in Reference [41] provides a detailed analysis of cache consistency in VNDN and shows that maintaining only the FRESHNESSPERIOD in the Data packet, as foreseen in vanilla NDN, is not enough to ensure consistency in the CS. Indeed, the FRESHNESSPERIOD does not indicate when the content has been generated, but only the time period it can remain in the CS since it has been cached. The value is also immutable and the cacher(s) cannot update it. In vehicular environments, especially when mobile nodes act as data mules, this behavior can create some major inconsistency effects, as demonstrated in the following example from Reference [41]. Let us consider a simple scenario where an RSU monitors the average speed on a certain road and produces Data packets named avgSpeed with FRESHNESSPERIOD $=60 \mathrm{~s}$. An NDN car A passing nearby requests the Data at time $t=0 \mathrm{~s}$ and it is allowed to cache it for $60 \mathrm{~s}$. Then, when $t=50 \mathrm{~s}$ another vehicle $\mathrm{B}$ asks for the same packet and receives it from A. By reading the FRESHNESSPERIOD, the packet could be stored in the CS of node B for $60 \mathrm{~s}$, but the actual residual lifetime of the packet is only $10 \mathrm{~s}$. If, at $t=70 \mathrm{~s}$, a vehicle $C$ receives the same packet from $\mathrm{B}$, the information is actually stale but $C$ does not have any means to understand this. Therefore, to avoid cache inconsistency and correctly compute the residual lifetime, a timestamp should be added in the Data packet header. If adding additional timing information is not possible, e.g., when data are provided by IoV sensors implementing low-rate and low-power protocols and there are limitations in the packet size, consumers can apply forecasting methods, like the one considered in Reference [50], to estimate the residual lifetime.

\subsection{Caching Policies}

The simplest caching scheme for VNDN conceived for transient contents is deployed in Reference [41]. The strategy, called Freshness-Driven Caching (FDC), distinguishes between short- and long- lasting contents and varies the caching probability depending on the residual lifetime (RL) of the received Data packet. The RL parameter of a packet $d_{k}$ is computed by considering the FRESHNESSPERIOD, the timestamp (additionally included by the source to avoid cache inconsistency) and the time instant $t$ when the packet $d_{k}$ is received, as follows: $R L\left(d_{k}\right)=$ timestamp $\left(d_{k}\right)+$ FreshnessPeriod $\left(d_{k}\right)-t$. If $R L\left(d_{k}\right)<0$ the packet is expired.

A dynamic threshold Th, used to distinguish long- or short-lasting contents, is obtained as the exponential weighted moving average (EWMA) of the residual lifetimes carried by the received packets. If the residual lifetime $R L\left(d_{k}\right)$ is greater than the threshold, the packet is cached with probability $p=1$; otherwise, it is cached with probability $p=\frac{R L\left(d_{k}\right)}{T h}$. The rationale behind the strategy is that, in presence of a uniform traffic distribution, as it would be in the case of some vehicular traffic, long-lasting contents have a higher chance to be requested during their lifetime. Therefore, long-lasting contents are always cached, while short-lasting contents are cached with a lower probability that tends to zero if the residual lifetime is going to expire.

The work in Reference [51], instead, defines a caching mechanism for RSUs only, based on service latency and content freshness. The authors clarify that the service latency shows how fast users get the contents after raising a request, while the freshness shows if the result is updated or not. The two factors are conflicting: to guarantee high data freshness requires frequent data exchanges that, in turn, create network congestion and increase the service latency. The proposed scheme, called Cache-Assisted Lazy Update and Delivery (CALUD), aims at balancing three factors: content update, delivery, and radio resource allocation. A Time Division Multiple Access (TDMA) scheme is implemented, 
where the RSU takes into account the received content requests and accommodates them in a First-In-First-Out (FIFO) fashion. To serve more users requesting the same information, downlink broadcast transmissions are used.

When vehicular contents are characterized by a skewed popularity distribution, like many Internet contents, popularity becomes an important caching decision metric that should be taken into account. Some works apply popularity-based caching at infrastructure nodes only, e.g., RSUs [52], while many others assume that also vehicles are able to estimate the popularity of contents, although the latter may largely change with the position of the nodes (i.e., vehicular contents have usually a specific geographical relevance) [53]. However, it is worth observing that, in presence of transient contents, the popularity metric should be matched with the lifetime information: caching contents that are going to expire immediately could be useless, and it may also force a high number of replacement operations.

This observation is taken into account by the Diversity-improved cAchiNg of popular Transient contEnts (DANTE) strategy in Reference [54], which defines a composite decision metric based on three content parameters, namely popularity, residual lifetime, and availability, as autonomously perceived by the vehicles in their neighborhood. A first target is to cache with high probability the popular long-lasting contents, which can likely serve more requests during their lifetime. Therefore, each vehicle maintains information of request rate and freshness of received contents. At the same time, however, DANTE aims to improve the cache diversity (and minimize the redundancy) by reducing the caching probability if contents are already available in the local neighborhood. Therefore, Data packets carry an hop count that is set to zero by the source (or cacher), and it is increased by one unit, hop by hop, at each receiver. The higher the hop count the lower the availability of the content in the neighborhood and, consequently, the higher should be the caching probability.

\subsection{Replacement Policies}

As discussed in Reference [55], replacement policies, like LRU or LFU, traditionally used in wired networks, could be not well suited for VANETs in certain scenarios. Indeed, the request pattern of vehicular contents can be driven by specific conditions, like vehicle mobility and geographical position or application priority and popularity. These parameters could highly influence the replacement policy. However, the majority of existing works consider the LRU scheme, due to its intrinsic simplicity; only very few ones specifically target cache replacement in VNDN and they almost unanimously do not refer to transient contents. For instance, the Popularity-based Content Caching (PopCC) policy in Reference [56] employs a Hidden Markov Model to predict the future popularity of contents and then caches the items in descending order of popularity. The last packet in the CS is the one with the lower popularity and the first candidate to be evicted. Popularity is also used in Reference [57] as a replacement metric, but in a cooperative fashion. There, vehicles are organized in clusters and the cluster-head, which maintains a central vision of request rate and hit ratio, decides which contents must be evicted.

To the best of our knowledge, only the two following works specifically target the replacement of transient contents. In particular, in Reference [58], vehicular contents are distinguished into three main classes: (i) safety information, such as emergency warnings messages, which expires quickly; (ii) road traffic information, which has a longer lifetime compared to safety contents; and (iii) comfort information, which has the longest lifetime. The CS of caching vehicles is then divided into three sections (called sub-CS), each one for a content class, thus guaranteeing that all types of vehicular traffic receive a proper quality of service (QoS) treatment. In particular, more space is provided to comfort traffic which is usually long-lived and larger in size, while less space is provided to safety traffic since it is usually small in size and highly transient. A TAG is included by the producer in the packet header to indicate the traffic class and guide the CS management. The replacement policy is based on a Content Popularity-Density metric that balances popularity and size of contents; it is computed by the producer and included in the header of the Data packets. When a vehicle receives a Data packet, it first checks the TAG and caches it if there is room 
in the related sub-CS. Otherwise, it extracts the popularity-density metric and compares it against the lower value associated with the cached items. If the newly received value is higher, then the existing cached item is replaced in favor of the new one.

The authors in Reference [55] present a Multi-Metric Cache Replacement policy $\left(\mathrm{M}^{2} \mathrm{CRP}\right)$ based on three peculiar factors: (i) freshness of the content, as carried in the FRESHNESSPERIOD field; (ii) frequency of the content requests, referred to the period the item has been cached in the CS; and (iii) distance between the current location of the vehicle and its position when it has originally cached the item. Intuitively, highly requested and fresh contents should be maintained in the CS. Moreover, since vehicular contents are usually location-based, they lose their relevance when the vehicle moves far away from the area they have been cached. Therefore, if the distance factor is high, the content has a high probability to be replaced.

When a new Data packet is received and the CS is full, $\mathrm{M}^{2} \mathrm{CRP}$ selects the candidate item to be replaced by computing a candidacy score for each content in the CS. A score function is defined that equally weights the three mentioned parameters, and the cached item with the lowest score is selected to be replaced.

\section{Insights and Research Perspectives}

Although some notable examples can be found in the literature, as reviewed in the previous sections, caching and replacement policies for transient contents in VNDN are still largely unexplored and there is room for further enhancements. Since vehicular traffic, applications, mobility conditions and nodes' density are highly heterogeneous, it is expected that there will be not a single reference policy but multiple ones will likely co-exist, depending on the context. As summarized in Table 2, the main parameters impacting caching and replacement are generally related to the content, e.g., lifetime, popularity. However, also node-related attributes can be taken into account, like geographical position and mobility pattern.

Moreover, transient contents caching also cover an important role in new and exciting research contexts, like the ones reported below.

Joint caching and processing of transient contents. In addition to the storage and communication resources, vehicles are provided with computing facilities that are generally underutilized. The groundbreaking Vehicular Cloud Computing (VCC) paradigm has been conceived to let vehicles offer joint caching and computing services on the road in a cooperative fashion [59]. Many works have already focused on aspects related to autonomous cloud formation and maintenance and optimal tasks allocation in traditional VANETs, while, in Reference [60], NDN has been recognized as a fundamental and effective content distribution architecture for VCC. In addition to data retrieval and in-network caching, NDN can be employed to directly enable in-network processing services [61]. According to such a new revolutionary vision, sometimes referred to as Named Function Networking (NFN) [62], consumers use Interest packets to request a named computing function to be applied over a named content and the network finds a node able to execute the task and return the result. Comprehensive studies evaluating NFN in vehicular environments and considering the joint caching and processing of transient contents, are still missing. The possibility of coupling Mobile Edge Computing (MEC) and VCC via NFN is also an interesting research direction, as outlined in Reference [63].

Caching transient contents in software defined vehicular networking. Software Defined Networking (SDN) is a new network paradigm that improves network management and performance by decoupling the control plane and the data plane. A centralized Controller with a global view of the network implements control plane functions, like optimized routing and security, and then instructs the network nodes. SDN has been applied in the context of vehicular networks to manage the highly dynamic communication and networking resources [64], while the work in Reference [65] has recognized the complementary features of SDN and NDN paradigms. On the one side, NDN can leverage SDN to simplify the network deployment and improve the routing decisions. On the other 
side, SDN can leverage the two NDN built-in features, stateful data plane and in-network caching, to further optimize the data delivery performance.

In the VNDN context, new functions could be introduced at the Controller to optimally coordinate the caching decisions of vehicular nodes. We encourage the community to consider this promising research aspect.

Improved transient content caching with Artificial Intelligence (AI) techniques. AIbased algorithms are a powerful means to improve network resource management and orchestration, even in presence of highly dynamic environments, like vehicular networks. By interacting with the environment and collecting feedback, they can improve the learning and forecasting capabilities of nodes while also solving complex optimization problems [66]. AI-based algorithms, if leveraged in the caching strategy, can help in predicting the popularity and the expected cache hit ratio of contents during their residual lifetime. A key deployment challenge in the NDN context is related to the fact that caching operations must be performed at line speed (during the forwarding process); therefore, decision algorithms should be simple and fast [67]. We can foresee a scenario where AI-algorithms are deployed off-line or in an asynchronous fashion and then the results are taken as input by the caching strategy. We encourage the research community to put efforts in this timely topic and explore the practical relevance of AI-driven caching strategies in real NDN implementations.

Table 2. Main decision parameters for caching and replacing transient contents in VNDN.

\begin{tabular}{|c|c|c|}
\hline Metric & Target & Reference Paper (s) \\
\hline Residual Lifetime & $\begin{array}{l}\text { To evict stale contents thus ensuring } \\
\text { cache consistency } \\
\text { To cache with higher probability } \\
\text { long-lasting contents } \\
\text { To replace with higher priority items } \\
\text { that are ready to expire }\end{array}$ & {$[41,51,54,55]$} \\
\hline Popularity & $\begin{array}{l}\text { - To cache with higher probability } \\
\text { popular contents } \\
\text { To replace with higher priority less } \\
\text { popular contents }\end{array}$ & {$[53-57]$} \\
\hline Distance & $\begin{array}{l}\text { - To replace with higher priority contents } \\
\text { cached in areas that are far away from } \\
\text { the current vehicle's position }\end{array}$ & {$[55]$} \\
\hline Availability & $\begin{array}{l}\text { - To cache with higher probability the } \\
\text { contents that are not available in the } \\
\text { local neighborhood }\end{array}$ & {$[54]$} \\
\hline Vehicular Content Class & $\begin{array}{l}\text { To differentiate the caching probability } \\
\text { and the local storage space depending } \\
\text { on the content class, which is } \\
\text { determined by size and lifetime } \\
\text { attributes }\end{array}$ & {$[58]$} \\
\hline
\end{tabular}

\section{Conclusions}

In this paper we provided a review of research works focusing on caching transient contents in VNDN. Our analysis also considered the role of transient contents in traditional (non-NDN-based) VANETs, when deploying safety and non-safety applications. Since vehicular applications are usually interested in timely and updated information, freshness is the most crucial parameter to be considered in caching decision and replacement strategies, but it is not the only one. If the contents exhibit a skewed popularity distribution, e.g., Zipflike, popularity also becomes an essential decision metric. 
Research in this field is still at its infancy, but we expect that the interest will grow fast in the next few years, thanks to increasing diffusion of IoT/IoV traffic and intelligent driving applications.

Funding: This research received no external funding.

Conflicts of Interest: The author declares no conflict of interest.

\section{Abbreviations}

The following abbreviations are used in this manuscript:

$\begin{array}{ll}\text { AI } & \text { Artificial Intelligence } \\ \text { AoI } & \text { Age of Information } \\ \text { CAM } & \text { Cooperative Awareness Messages } \\ \text { CEE } & \text { Cache Everything Everywhere } \\ \text { CS } & \text { Content Store } \\ \text { ETSI } & \text { European Telecommunications Standards Institute } \\ \text { FIB } & \text { Forwarding Information Base } \\ \text { FIFO } & \text { First In First Out } \\ \text { GPS } & \text { Global Positioning System } \\ \text { ICN } & \text { Information Centric Networking } \\ \text { IoT } & \text { Internet of Things } \\ \text { IoV } & \text { Internet of Vehicles } \\ \text { ITS } & \text { Intelligent Transportation System } \\ \text { LFU } & \text { Least Frequently Used } \\ \text { LRU } & \text { Least Recently Used } \\ \text { MAC } & \text { Medium Access Control } \\ \text { NDN } & \text { Named Data Networking } \\ \text { PIT } & \text { Pending Interest Table } \\ \text { RSU } & \text { Road Side Unit } \\ \text { SDN } & \text { Software Defined Networking } \\ \text { VANET } & \text { Vehicular Ad Hoc Network } \\ \text { VCC } & \text { Vehicular Cloud Computing } \\ \text { V2I } & \text { Vehicle To Infrastructure } \\ \text { V2V } & \text { Vehicle To Vehicle } \\ \text { VNDN } & \text { Vehicular NDN } \\ & \end{array}$

\section{References}

1. Zhang, L.; Afanasyev, A.; Burke, J.; Jacobson, V.; Claffy, K.; Crowley, P.; Papadopoulos, C.; Wang, L.; Zhang, B. Named data networking. ACM SIGCOMM Comput. Commun. Rev. 2014, 44, 66-73. [CrossRef]

2. Meisel, M.; Pappas, V.; Zhang, L. Ad hoc networking via named data. In Proceedings of the Fifth ACM International Workshop on Mobility in the Evolving Internet Architecture, Chicago, IL, USA, 20-24 September 2010; pp. 3-8.

3. Amadeo, M.; Campolo, C.; Molinaro, A. CRoWN: Content-Centric Networking in Vehicular Ad Hoc Networks. IEEE Commun. Lett. 2012, 16, 1380-1383. [CrossRef]

4. Grassi, G.; Pesavento, D.; Pau, G.; Vuyyuru, R.; Wakikawa, R.; Zhang, L. VANET via Named Data Networking. In Proceedings of the 2014 IEEE Conference on Computer Communications Workshops, Toronto, ON, Canada, 27 April-2 May 2014; pp. 410-415.

5. Yaqub, M.A.; Ahmed, S.H.; Bouk, S.H.; Kim, D. Interest forwarding in vehicular information centric networks: A survey. In Proceedings of the 31st Annual ACM Symposium on Applied Computing, Pisa, Italy, 4-8 April 2016; pp. 724-729.

6. Borrego, C.; Amadeo, M.; Molinaro, A.; Mendes, P.; Sofia, R.C.; Magaia, N.; Borrell, J. Forwarding in Opportunistic InformationCentric Networks: An Optimal Stopping Approach. IEEE Commun. Mag. 2020, 58, 56-61. [CrossRef]

7. Ahmed, S.H.; Bouk, S.H.; Yaqub, M.A.; Kim, D.; Song, H.; Lloret, J. CODIE: Controlled data and interest evaluation in vehicular named data networks. IEEE Trans. Veh. Technol. 2016, 65, 3954-3963. [CrossRef]

8. Huang, W.; Song, T.; Yang, Y.; Zhang, Y. Cluster-based selective cooperative caching strategy in vehicular named data networking. In Proceedings of the IEEE International Conference on Hot Information-Centric Networking (HotICN), Shenzhen, China, 15-17 August 2018; pp. 7-12.

9. Yao, L.; Chen, A.; Deng, J.; Wang, J.; Wu, G. A cooperative caching scheme based on mobility prediction in vehicular content centric networks. IEEE Trans. Veh. Technol. 2017, 67, 5435-5444. [CrossRef]

10. Zhang, J.; Letaief, K.B. Mobile Edge Intelligence and computing for the Internet of Vehicles. Proc. IEEE 2019, 108, $246-261$. [CrossRef] 
11. Bouk, S.H.; Ahmed, S.H.; Kim, D. Vehicular content centric network (VCCN) a survey and research challenges. In Proceedings of the 30th Annual ACM Symposium on Applied Computing, Salamanca, Spain, 13-17 April 2015; pp. 695-700.

12. Amadeo, M.; Campolo, C.; Molinaro, A. Information-centric networking for connected vehicles: A survey and future perspectives. IEEE Commun. Mag. 2016, 54, 98-104. [CrossRef]

13. Ahed, K.; Benamar, M.; Lahcen, A.A.; El Ouazzani, R. Forwarding Strategies in Vehicular Named Data Networks: A survey. J. King Saud Univ. Comput. Inf. Sci. 2020. [CrossRef]

14. Khelifi, H.; Luo, S.; Nour, B.; Moungla, H.; Faheem, Y.; Hussain, R.; Ksentini, A. Named data networking in vehicular ad hoc networks: State-of-the-art and challenges. IEEE Commun. Surv. Tutor. 2019, 22, 320-351. [CrossRef]

15. Chen, C.; Wang, C.; Qiu, T.; Atiquzzaman, M.; Wu, D.O. Caching in vehicular named data networking: Architecture, schemes and future directions. IEEE Commun. Surv. Tutor. 2020, 22, 2378-2407. [CrossRef]

16. Amadeo, M.; Campolo, C.; Molinaro, A.; Harri, J.; Rothenberg, C.E.; Vinel, A. Enhancing the 3GPP V2X architecture with information-centric networking. Future Internet 2019, 11, 199. [CrossRef]

17. Ahmed, S.H.; Bouk, S.H.; Yaqub, M.A.; Kim, D.; Gerla, M. CONET: Controlled data packets propagation in vehicular named data networks. In Proceedings of the 2016 13th IEEE Annual Consumer Communications \& Networking Conference, Las Vegas, NV, USA, 9-12 January 2016; pp. 620-625.

18. Amadeo, M.; Campolo, C.; Molinaro, A. Enhancing content-centric networking for vehicular environments. Comput. Netw. 2013, 57, 3222-3234. [CrossRef]

19. Grassi, G.; Pesavento, D.; Pau, G.; Zhang, L.; Fdida, S. Navigo: Interest forwarding by geolocations in vehicular named data networking. In Proceedings of the 2015 IEEE 16th International Symposium on A World of Wireless, Mobile and Multimedia Networks, Boston, MA, USA, 14-17 June 2015; pp. 1-10.

20. Wahid, A.; Shah, M.A.; Qureshi, F.F.; Maryam, H.; Iqbal, R.; Chang, V. Big data analytics for mitigating broadcast storm in vehicular content centric networks. Future Gener. Comput. Syst. 2018, 86, 1301-1320. [CrossRef]

21. Tarnoi, S.; Suksomboon, K.; Kumwilaisak, W.; Ji, Y. Performance of Probabilistic Caching and Cache Replacement Policies for Content-Centric Networks. In Proceedings of the 39th Annual IEEE Conference on Local Computer Networks, Edmonton, AB, Canada, 8-11 September 2014; pp. 99-106.

22. Modesto, F.M.; Boukerche, A. An Analysis of Caching in Information-Centric Vehicular Networks. In Proceedings of the 2017 IEEE International Conference on Communications, Paris, France, 21-25 May 2017; pp. 1-6.

23. Kaul, S.; Gruteser, M.; Rai, V.; Kenney, J. Minimizing age of information in vehicular networks. In Proceedings of the IEEE Communications Society Conference on Sensor, Mesh and Ad Hoc Communications and Networks, Salt Lake City, UT, USA, 27-30 June 2011; pp. 350-358.

24. Lyamin, N.; Vinel, A.; Jonsson, M.; Bellalta, B. Cooperative awareness in VANETs: On ETSI EN 302 637-2 performance. IEEE Trans. Veh. Technol. 2017, 67, 17-28. [CrossRef]

25. Zhou, B.; Saad, W. Joint status sampling and updating for minimizing age of information in the Internet of Things. IEEE Trans. Commun. 2019, 67, 7468-7482. [CrossRef]

26. Lochert, C.; Scheuermann, B.; Caliskan, M.; Mauve, M. The feasibility of information dissemination in vehicular ad-hoc networks In Proceedings of the Conference on Wireless on Demand Network Systems and Services, Obergurgl, Tyrol, Austria, 24-26 January 2007; pp. 92-99.

27. Caliskan, M.; Graupner, D.; Mauve, M. Decentralized discovery of free parking places. In Proceedings of the 3rd International Workshop on Vehicular Ad Hoc Networks, Los Angeles, CA, USA, 29 September 2006; pp. 30-39.

28. Baiocchi, A.; Turcanu, I. A model for the optimization of beacon message age-of-information in a VANET. In Proceedings of the 2017 29th International Teletraffic Congress (ITC 29), Genoa, Italy, 4-8 September 2017; Volume 1, pp. 108-116.

29. Kaul, S.; Yates, R.; Gruteser, M. Real-time status: How often should one update? In Proceedings of the IEEE INFOCOM, Orlando, FL, USA, 25-30 March 2012; pp. 2731-2735.

30. Costa, M.; Codreanu, M.; Ephremides, A. On the age of information in status update systems with packet management. IEEE Trans. Inf. Theory 2016, 62, 1897-1910. [CrossRef]

31. Abd-Elmagid, M.A.; Pappas, N.; Dhillon, H.S. On the Role of Age of Information in the Internet of Things. IEEE Commun. Mag. 2019, 57, 72-77. [CrossRef]

32. Abdel-Aziz, M.K.; Samarakoon, S.; Liu, C.F.; Bennis, M.; Saad, W. Optimized age of information tail for ultra-reliable low-latency communications in vehicular networks. IEEE Trans. Commun. 2019, 68, 1911-1924. [CrossRef]

33. Ang, L.M.; Seng, K.P.; Ijemaru, G.K.; Zungeru, A.M. Deployment of IoV for smart cities: Applications, architecture, and challenges. IEEE Access 2018, 7, 6473-6492. [CrossRef]

34. Malandrino, F.; Casetti, C.; Chiasserini, C.F.; Sommer, C.; Dressler, F. The role of parked cars in content downloading for vehicular networks. IEEE Trans. Veh. Technol. 2014, 63, 4606-4617. [CrossRef]

35. Fiore, M.; Casetti, C.; Chiasserini, C.F. Caching strategies based on information density estimation in wireless ad hoc networks. IEEE Trans. Veh. Technol. 2011, 60, 2194-2208. [CrossRef]

36. Ayaida, M.; Barhoumi, M.; Fouchal, H.; Ghamri-Doudane, Y.; Afilal, L. HHLS: A hybrid routing technique for VANETs. In Proceedings of the IEEE Global Communications Conference (GLOBECOM), Anaheim, CA, USA, 3-7 December 2012; pp. 44-48.

37. Nzouonta, J.; Rajgure, N.; Wang, G.; Borcea, C. VANET routing on city roads using real-time vehicular traffic information. IEEE Trans. Veh. Technol. 2009, 58, 3609-3626. [CrossRef] 
38. Cao, J.; Zhang, Y.; Cao, G.; Xie, L. Data consistency for cooperative caching in mobile environments. IEEE Comput. 2007, 40, 60-66. [CrossRef]

39. Lim, S.; Yu, C.; Das, C.R. Cooperative cache invalidation strategies for internet-based vehicular ad hoc networks. In Proceedings of the 18th IEEE International Conference on Computer Communications and Networks, San Francisco, CA, USA, 3-6 August 2009; pp. 1-6.

40. Kam, C.; Kompella, S.; Nguyen, G.D.; Wieselthier, J.E.; Ephremides, A. Information freshness and popularity in mobile caching. In Proceedings of the IEEE International Symposium on Information Theory (ISIT), Aachen, Germany, 25-30 June 2017; pp. 136-140.

41. Amadeo, M.; Campolo, C.; Ruggeri, G.; Lia, G.; Molinaro, A. Caching Transient Contents in Vehicular Named Data Networking: A Performance Analysis. Sensors 2020, 20, 1985. [CrossRef]

42. Baccelli, E.; Mehlis, C.; Hahm, O.; Schmidt, T.C.; Wählisch, M. Information centric networking in the IoT: Experiments with NDN in the wild. In Proceedings of the 1st ACM Conference on Information-Centric Networking, Paris, France, 24-26 September 2014; pp. 77-86.

43. Vural, S.; Wang, N.; Navaratnam, P.; Tafazolli, R. Caching transient data in internet content routers. IEEE/ACM Trans. Netw. 2016, 25, 1048-1061. [CrossRef]

44. Hail, M.A.; Amadeo, M.; Molinaro, A.; Fischer, S. Caching in Named Data Networking for the Wireless Internet of Things. In Proceedings of the 2015 International Conference on Recent Advances in Internet of Things (RIoT), Singapore, 7-9 April 2015; pp. 1-6.

45. Hahm, O.; Baccelli, E.; Schmidt, T.C.; Wählisch, M.; Adjih, C.; Massoulié, L. Low-power internet of things with NDN \& cooperative caching. In Proceedings of the ACM Conference on Information-Centric Networking, Berlin, Germany, 26-28 September 2017; pp. 98-108.

46. Amadeo, M.; Ruggeri, G.; Campolo, C.; Molinaro, A.; Mangiullo, G. Caching Popular and Fresh IoT Contents at the Edge via Named Data Networking. In Proceedings of the IEEE INFOCOM WKSHPS, Toronto, ON, Canada, 6-9 July 2020 ; pp. 610-615.

47. Zhang, Z.; Lung, C.H.; Lambadaris, I.; St-Hilaire, M. IoT data lifetime-based cooperative caching scheme for ICN-IoT networks. In Proceedings of the 2018 IEEE International Conference on Communications (ICC), Kansas City, MO, USA, 20-24 May 2018; pp. 1-7.

48. Ong, M.D.; Chen, M.; Taleb, T.; Wang, X.; Leung, V. FGPC: Fine-Grained Popularity-based Caching Design for Content Centric Networking. In Proceedings of the ACM International Conference on Modeling, Analysis and Simulation of Wireless and Mobile Systems, Montreal, QC, Canada, 21-26 September 2014; pp. 295-302.

49. Abdullahi, I.; Arif, S.; Hassan, S. Survey on caching approaches in information centric networking. J. Netw. Comput. Appl. 2015, 56, 48-59. [CrossRef]

50. Meddeb, M.; Dhraief, A.; Belghith, A.; Monteil, T.; Drira, K.; Mathkour, H. Least fresh first cache replacement policy for NDN-based IoT networks. Pervasive Mob. Comput. 2019, 52, 60-70. [CrossRef]

51. Zhang, S.; Li, J.; Luo, H.; Gao, J.; Zhao, L.; Shen, X.S. Towards fresh and low-latency content delivery in vehicular networks: An edge caching aspect. In Proceedings of the IEEE International Conference on Wireless Communications and Signal Processing (WCSP), Hangzhou, China, 18-20 October 2018; pp. 1-6.

52. Ding, R.; Wang, T.; Song, L.; Han, Z.; Wu, J. Roadside-Unit Caching in Vehicular Ad Hoc Networks for Efficient Popular Content Delivery. In Proceedings of the IEEE Wireless Communications and Networking Conference (WCNC), New Orleans, LA, USA, 9-12 March 2015; pp. 1207-1212.

53. Quan, W.; Liu, Y.; Jiang, X.; Guan, J. Intelligent popularity-aware content caching and retrieving in highway vehicular networks. EURASIP J. Wirel. Commun. Netw. 2016, 2016, 1-10. [CrossRef]

54. Amadeo, M.; Ruggeri, G.; Campolo, C.; Molinaro, A. Diversity-improved caching of popular transient contents in Vehicular Named Data Networking. Comput. Netw. 2020, 184, 107625. [CrossRef]

55. Ostrovskaya, S.; Surnin, O.; Hussain, R.; Bouk, S.H.; Lee, J.; Mehran, N.; Ahmed, S.H.; Benslimane, A. Towards Multi-metric Cache Replacement Policies in Vehicular Named Data Networks. In Proceedings of the IEEE 29th Annual International Symposium on Personal, Indoor and Mobile Radio Communications (PIMRC), Bologna, Italy, 9-12 September 2018; pp. 1-7.

56. Yao, L.; Wang, Y.; Xia, Q.; Xu, R. Popularity Prediction Caching Using Hidden Markov Model for Vehicular Content Centric Networks. In Proceedings of the 2019 20th IEEE International Conference on Mobile Data Management (MDM), Hong Kong, China, 10-13 June 2019; pp. 533-538.

57. Chootong, S.; Thaenthong, J. Cache replacement mechanism with content popularity for vehicular content-centric networks (vccn). In Proceedings of the IEEE International Joint Conference on Computer Science and Software Engineering (JCSSE), NakhonSiThammarat, Thailand, 12-14 July 2017; pp. 1-6.

58. Khelifi, H.; Luo, S.; Nour, B.; Moungla, H. A QoS-aware cache replacement policy for Vehicular Named Data Networks. In Proceedings of the IEEE Global Communications Conference (GLOBECOM), Waikoloa, HI, USA, 9-13 December 2019; pp. 1-6.

59. Whaiduzzaman, M.; Sookhak, M.; Gani, A.; Buyya, R. A survey on vehicular cloud computing. J. Netw. Comput. Appl. 2014, 40, 325-344. [CrossRef]

60. Gerla, M.; Lee, E.K.; Pau, G.; Lee, U. Internet of vehicles: From intelligent grid to autonomous cars and vehicular clouds. In Proceedings of the IEEE World Forum on Internet of Things (WF-IoT), Seoul, Korea, 6-8 March 2014; pp. 241-246. 
61. Amadeo, M.; Ruggeri, G.; Campolo, C.; Molinaro, A. Iot services allocation at the edge via named data networking: From optimal bounds to practical design. IEEE Trans. Netw. Serv. Manag. 2019, 16, 661-674. [CrossRef]

62. Tschudin, C.; Sifalakis, M. Named functions and cached computations. In Proceedings of the IEEE 11th Consumer Communications and Networking Conference (CCNC), Las Vegas, NV, USA, 10-13 January 2014; pp. 851-857.

63. Grewe, D.; Wagner, M.; Arumaithurai, M.; Psaras, I.; Kutscher, D. Information-centric mobile edge computing for connected vehicle environments: Challenges and research directions. In Proceedings of the Workshop on Mobile Edge Communications, Los Angeles, CA, USA, 21 August 2017; pp. 7-12.

64. Quan, W.; Liu, Y.; Zhang, H.; Yu, S. Enhancing crowd collaborations for software defined vehicular networks. IEEE Commun. Mag. 2017, 55, 80-86. [CrossRef]

65. Zhang, Q.Y.; Wang, X.W.; Huang, M.; Li, K.Q.; Das, S.K. Software defined networking meets information centric networking: A survey. IEEE Access 2018, 6, 39547-39563. [CrossRef]

66. Dai, Y.; Xu, D.; Maharjan, S.; Qiao, G.; Zhang, Y. Artificial intelligence empowered edge computing and caching for internet of vehicles. IEEE Wirel. Commun. 2019, 26, 12-18. [CrossRef]

67. Rossi, D.; Rossini, G. Caching Performance of Content Centric Networks under Multi-Path Routing (and More). Technical Report, Telecom ParisTech; 2011; pp. 1-6. Available online: https://perso.telecom-paristech.fr/drossi/paper/rossi11ccn-techrep1.pdf (accessed on 8 February 2021). 